\title{
Expert*innenbewertung \\ der Methoden zum \\ Fledermausmonitoring bei \\ Windkraftvorhaben
}

\section{Expert evaluations of methods \\ used for monitoring bats \\ during wind turbine projects}

Christian C. Voigt, Manuel Roeleke, Olga Heim, Linn S. Lehnert, Marcus Fritze und Oliver Lindecke

\section{Zusammenfassung}

Die Erfassung des Artvorkommens und der Aktivität von Fledermäusen im Rahmen von Genehmigungsverfahren für Windenergieanlagen (WEA) beruht auf der legalen Notwendigkeit, das gesetzlich verankerte Störungsund Tötungsverbot geschützter Arten umzusetzen. In einer Internetumfrage baten wir Fachexpert*innen um eine Einschätzung der Eignung und Praxistauglichkeit von Methoden, die gegenwärtig für die Begleituntersuchung von

C. C. Voigt $(\bowtie) \cdot$ M. Roeleke · O. Heim · L. S. Lehnert · M. Fritze · O. Lindecke

Abteilung Evolutionäre Ökologie, Leibniz-Institut für Zoo- und Wildtierforschung,

Berlin, Deutschland

E-Mail: voigt@izw-berlin.de

M. Roeleke

E-Mail: roeleke@izw-berlin.de

O. Heim

E-Mail: heim@izw-berlin.de

L. S. Lehnert

E-Mail: lehnert@izw-berlin.de

M. Fritze

E-Mail: fritze@izw-berlin.de

O. Lindecke

E-Mail: lindecke@izw-berlin.de 
Fledermäusen eingesetzt werden. Der Netzfang und die manuelle sowie automatische Erfassung der akustischen Aktivität von Fledermäusen in Bodennähe betrachteten die Fachexpert*innen als ungeeignet oder bedingt geeignet, um das Schlagrisiko für Fledermäuse an WEA bewerten zu können. Dies galt vor allem für Standorte im Offenland wie zum Beispiel auf Agrarflächen. Die automatische Erfassung der akustischen Aktivität von Fledermäusen in Gondelhöhe (Gondelmessung) wurde mehrheitlich, vor allem für den Zeitraum der Herbstmigration, als geeignet erachtet. Behördenvertreter*innen und Fachgutachter*innen bewerteten die Gondelmessung als wenig praxistauglich, aber prinzipiell geeignet. Der Netzfang wurde für Waldstandorte und speziell für die Wochenstubenzeit als geeignete Methode zur Erfassung der lokalen Fledermausaktivität beurteilt. Die Suche nach Quartierstandorten mittels Radiotelemetrie wurde besonders für Waldstandorte als geeignet bewertet. Fachgutachter*innen gaben häufiger als Behördenvertreter*innen an, dass sie Diskrepanzen zwischen der Gondelmessung und der Schlagopferzahl beobachten konnten. Die Fachexpert*innen waren sich uneinig darüber, ob die Schätzwerte für Schlagopfer mittels spezieller Hochrechnungen der Realität entsprechen. Nur etwa jeder fünfte Befragte gab an, dass diese Hochrechnung realistische Zahlen liefere. Die Mehrheit der Fachexpert*innen vermutete eine Unterschätzung der tatsächlichen Schlagopferzahl. Die Ergebnisse unserer Umfrage zeigen, dass einige im Rahmen von Genehmigungsverfahren praktizierte Methoden von den Fachexpert*innen hinterfragt und deren Verlässlichkeit unter bestimmten Bedingungen kritisiert werden. Insbesondere schließen wir aus der Auswertung, dass die prinzipiell als geeignet bewertete akustische Aktivitätsmessung in Gondelhöhe mit Schlagopfersuchen an den betreffenden WEA kombiniert werden sollte, um die Unsicherheiten der einzeln angewandten Methoden zu reduzieren.

\section{Summary}

Owing to the legal protection of bats and their habitats, it is mandatory to monitor the presence and activity of bats as part of environmental impact assessments during the planning process of wind turbine projects. During an internet-based survey, we asked involved experts to judge the practicability and feasibility of methods, which are currently applied to monitor bats. The mist-netting of bats and both automated and non-automated acoustic recordings at ground level were judged unsuitable or conditionally suitable to assess the mortality risk of bats at wind turbines. This was considered particularly true for open areas, such as farmland. The majority of experts considered the automated acoustic monitoring of bat activity at nacelle height (nacelle monitoring) as suitable in general, but not necessarily practical. The mist-netting of bats was judged suitable to document the activity of bats at forested sites, particularly during the maternity period. Consultants more so than members 
of the conservation agencies reported discrepancies between the acoustic recording of bats at nacelle height and the number of documented fatalities. The experts disagreed on whether or not the estimated number of killed bats was representative for the true number of killed bats at wind turbines. Only about one-fifth of responding experts reported that the estimated number of killed bats matched with true fatality rates. Indeed, the majority of respondents suggested that the estimated number of killed bats falls below the true number of killed bats at wind turbines. The results of our survey show that some of the methods applied as part of the environmental impact assessments procedure are scrutinized by experts. Particularly, experts questioned the validity of some of the widely applied methods for specific conditions. Overall, we conclude that the acoustic monitoring of bats at nacelle height should be combined with carcass searches to reduce the uncertainties involved in using only one of the aforementioned methods.

\subsection{Einleitung}

Bis zum Jahr 2050 plant die Bundesregierung eine vollständige Abkehr von fossilen und nuklearen Energieträgern und einen flächendeckenden Ausbau der Energieproduktion aus erneuerbaren Energieträgern wie Solarenergie, Biogas, Wind- und Wasserkraft. Das primäre Ziel dabei ist, die Kohlendioxidemission, die durch die Verbrennung von fossilen Brennstoffen entsteht, aus Klimaschutzgründen zu reduzieren und die gefährliche Energieproduktion aus nuklearen Energieträgern mit dem einhergehenden ungelösten Problem der Endlagerung zu stoppen (EEG 2017). Auch weltweit sind viele Länder bestrebt, die Energieproduktion aus erneuerbaren Energieträgern auszubauen, um die voranschreitende Erderwärmung einzudämmen. Auf der UN-Klimakonferenz in Paris 2015 haben sich deshalb mehr als 150 Länder dazu verpflichtet, die Weltwirtschaft und damit auch die Energieproduktion auf "klimafreundliche Weise“ zu verändern. In Deutschland werden momentan 29.213 Windenergieanlagen (WEA) mit einer kumulativen Leistung von $52.931 \mathrm{MW}$ an Land betrieben (Stand Dezember 2018; Deutsche WindGuard 2019). Aufgrund des großen Raumbedarfs, der durch die stetig wachsende Anzahl an WEA entsteht, kommt es vermehrt zu Interessenskonflikten in der Flächennutzung. Besonders im Fokus steht dabei das sogenannte Grün-Grün-Dilemma - ein Konflikt zwischen Klima- und Artenschutz (Voigt 2016; Voigt et al. 2019). Die vermehrten Funde von toten und verletzten Großvögeln unter WEA führten seit dem Bau der ersten WEA zu einer erhöhten Aufmerksamkeit gegenüber potenziellen Gefahren für Vögel. Um die Jahrtausendwende wurden durch das Auffinden von Fledermausschlagopfern zunehmend auch negative Auswirkungen auf Fledermäuse in Deutschland sowie die Populationen der Nachbarländer deutlich (Bach 2001; Dürr 2002; Dürr und Bach 2004; Voigt et al. 2012, 2015, 2016; Lehnert et al. 2014; Zahn et al. 2014). Diese reichen von Habitatverlust bis zur Tötung von Individuen durch direkten Schlag an Rotorblättern oder Verletzungen der inneren Organe durch abrupte 
Luftdruckunterschiede im Rotorbereich (Barotrauma; Baerwald et al. 2008; Voigt et al. 2015). Aufgrund ihres Schutzstatus in internationalen Konventionen (EU-Fauna-Flora-Habitat-Richtlinie, UN-Konvention zum Schutz migrierender Arten UNEP/EUROBATS) sowie nach nationalem Recht (Bundesnaturschutzgesetz, BNatSchG) zählen Fledermäuse in Deutschland zu den besonders und streng geschützten Arten. Gemäß $\S 44 \mathrm{BNatSchG}$ ist es deshalb verboten, Fledermäuse zu stören, zu töten oder deren Lebensstätten zu zerstören. Um den Zielen des Gesetzes zur Förderung der erneuerbaren Energien (EEG 2017) gerecht zu werden und gleichzeitig die Belange des BNatSchG einzuhalten, ist es notwendig, in sogenannten Artenschutzgutachten zu prüfen, ob beim Bau und Betrieb von WEA am jeweiligen Standort ein Verstoß gegen das BNatSchG vorliegt. Konkret wird untersucht, inwieweit Vögel und Fledermäuse im Gebiet vorkommen und ob sie durch den Bau oder den Betrieb der WEA im Sinne des §44 BNatSchG erheblich beeinträchtigt werden. Ist ein Verstoß absehbar, müssen Vermeidungsoder Verminderungsmaßnahmen geplant werden, damit eine Genehmigung erteilt werden kann.

Je nach Tierart kommen bei der Erfassung unterschiedliche Methoden zum Einsatz. Bei der Erfassung von Fledermäusen werden typischerweise bioakustische Empfängergeräte verwendet und Netzfänge durchgeführt. Besenderte Tiere werden zudem gegebenenfalls mittels Radiotelemetrie verfolgt und lokalisiert. Umfangreiche Methodenempfehlungen zur Untersuchung und zur Reduktion des Kollisionsrisikos von Fledermäusen wurden bereits 2011 von Brinkmann zusammengestellt (Brinkmann 2011). Das benötigte Methodenrepertoire muss sich jedoch einer dynamisch entwickelnden Situation anpassen. Zum einen werden die WEA-Typen zunehmend in Bau- und Betriebsweise (z. B. Gondelhöhe, Rotordurchmesser und Umdrehungsgeschwindigkeit) diverser und größer (Deutsche WindGuard 2019). Zum anderen wird die räumliche Situation, in denen WEA errichtet werden, immer vielfältiger. So umfassen die beplanten Standorte aufgrund der zunehmenden Flächenverknappung mehr Landschaftstypen als in den Anfangsjahren, in denen hauptsächlich landwirtschaftliche Nutzflächen betroffen waren (Deutsche WindGuard 2019). Seit 2010 kommt es zum Beispiel vermehrt zu Genehmigungsvorhaben in Forst- und Waldgebieten (80\% Zuwachs; FA Wind 2018), sowie zum Ausbau in Naturparken und FFH-Gebieten, als auch der Errichtung von WEA am Rand von streng geschützten Habitaten wie Naturschutzgebieten und Nationalparks. Ende 2017 ist bundesweit die Zahl von WEA im Wald auf ungefähr 1850 beziffert worden (FA Wind 2018). Zudem werden WEA heutzutage in unterschiedlichen Geländetypen installiert (z. B. Uferbereiche, Flachland, Täler, Höhenzüge und Plateaus). Diese sich weiter entwickelnde Situation erfordert eine an die jeweiligen Vorhaben angepasste Erfassungsmethodik, um potenziell negative Auswirkungen auf lokale sowie wandernde Fledermäuse vorherzusagen. Hurst et al. (2015) erarbeiteten speziell für Windkraftprojekte in Waldgebieten eine Synopsis der bundeslandspezifischen Handlungsempfehlungen. Unabhängig davon, ob WEA-Projekte im Wald oder im Offenland geplant werden, gibt es generelle Kritiken an den Methoden und deren Aussagekraft zur Abschätzung der Gefährdung von Fledermäusen. Während zum 
Beispiel Lintott et al. (2016) die Aussagekraft der Voruntersuchung am geplanten WEA-Standort infrage stellten, kritisierten Lindemann et al. (2018) vor allem die Verlässlichkeit der Ergebnisse von bioakustischen Aufnahmen in Gondelhöhe, die eine erhebliche Rolle bei der nachträglichen Untersuchung an den bereits gebauten WEA spielen. Eine Qualitätsprüfung von 156 Fledermausgutachten aus mehreren Bundesländern zeigte zudem, dass methodische Vorgaben weder einheitlich noch vollumfänglich eingehalten werden und deshalb eine Standardisierung gutachterlicher Methoden sowie deren Kontrolle dringend erforderlich sind (Gebhard et al. 2016).

Um während des Planungsprozesses für alle Interessensgruppen Rechtssicherheit zu schaffen und bundesweit vergleichbare Planungsverfahren zu gewährleisten, wären bundesweit einheitliche, standardisierte Methoden, welche die jeweils regional unterschiedlichen Standorte und Betriebsweisen der WEA berücksichtigen, notwendig. Allerdings existieren derzeit nur bundeslandspezifische Leitfäden. Diese unterscheiden sich teilweise stark in ihren Forderungen und Empfehlungen zur Erfassungsmethodik, da sie zu unterschiedlichen Zeitpunkten erstellt worden sind. Während ältere Leitfäden, wie z. B. der Windenergieerlass in Brandenburg (MLUL 2011) noch vor dem Erscheinen des Standardwerkes von Brinkmann et al. (2011) erstellt wurden, stammen die während der Tagung „Evidenzbasierter Fledermausschutz bei Windkraftvorhaben“ (Leibniz-IZW Berlin, März 2019) vorgestellten aktuellen Leitfäden aus Thüringen (Dietz et al. 2015) und Sachsen-Anhalt (MULE 2018) aus den Jahren 2015 bzw. 2018. Daraus ist abzuleiten, dass die in die Genehmigungsverfahren involvierten Personen (Fachgutachter*innen, Vertreter*innen der Behörden sowie des Windenergiesektors und zum Teil auch Umweltverbände und Wissenschaftler) mit unterschiedlichen Methoden, die sich aus den Empfehlungen der Leitfäden ergeben, arbeiten.

Die vorliegende bundesweite Expert*innenbefragung diente dazu, die derzeit angewandten Methoden anhand von Expert*innenmeinungen zu bewerten. Die Ergebnisse sollen dazu dienen, die besten Methoden für die spezifischen Situationen an den jeweiligen WEA-Standorten zu identifizieren und das jeweilige Methodenrepertoire nach dem Best-Practice-Prinzip darzustellen.

\subsection{Material und Methoden}

Unter Verwendung der Online-Umfragesoftware SurveyGizmo (https://www. surveygizmo.eu/) erstellten wir einen Fragebogen zum Thema „Vereinbarkeit von Fledermausschutz und Windenergieausbau in Deutschland“. Dieser bestand aus 50 Fragen bzw. Kommentaroptionen. Der Link dazu wurde per E-Mail an ungefähr 1000 Personen und Institutionen versandt, die sich professionell mit Fledermäusen und/oder Windkraft beschäftigen (Anhang Frage 2; Fritze et al. 2019). Zusätzlich riefen wir zur Weiterleitung an interessierte Personen auf. Die Teilnahme an der Umfrage war vom 03.05.2016 bis zum 27.05.2016 möglich. Zur Auswertung fassten wir folgende Expertengruppen zusammen: Behördenvertreter*innen, Fachgutachter*innen (Landschaftsplaner*innen und Fledermaus-Fachgutachter*innen), 
Vertreter*innen einer Umweltschutzorganisation (Angestellte sowie ehrenamtlich Tätige), Vertreter*innen des Windenergiesektors (Angestellte und Affiliierte von Windkraftkonzernen, Betreiber, Projektierer, Jurist*innen) und Wissenschaftler*innen (Wissenschaftler*innen im Themenfeld Fledermäuse, Wissenschaftler*innen im Themenfeld Windkraft). Personen anderer Berufsgruppen konnten sich unter der Kategorie Sonstige eintragen und an der Umfrage teilnehmen. Sofern möglich, integrierten wir diese nach Rücksprache in die entsprechende Expert*innengruppen. Elf Antwortbögen konnten nicht zugeordnet werden und wurden folglich nicht ausgewertet. Der in drei Blöcke unterteilte Fragebogen beinhaltete neben allgemeinen Fragen zur Charakterisierung der Expert*innengruppen vor allem Aspekte des Naturschutzrechts und der Erfassungsmethodik (markierte Fragen im Anhang). Zwei der Umfrageblöcke, die sich mit Aspekten des Genehmigungsverfahrens sowie mit dem Thema Artenschutz bzw. Fledermausschutz im Windenergieausbau beschäftigten, sind bereits an anderer Stelle publiziert (Fritze et al. 2019; Kap. 8). Bei den meisten Fragen baten wir um eine Bewertung von 1 (sehr schlecht) bis 5 (sehr gut). Im Folgenden stellen wir Antworten zu den Aspekten der Erfassungsmethodik vor, das heißt speziell zum Thema Netzfang, Radiotelemetrie, Detektorbegehung, automatisierte akustische Begleituntersuchung in Bodennähe und in Gondelhöhe (im Folgenden Gondelmessung genannt) sowie Schlagopfersuche. Aufgrund des hohen Rücklaufs seitens der Behördenvertreter*innen, Fachgutachter*innen und Vertreter*innen von Umweltschutzorganisationen wurden besonders für diese Gruppen statistische Auswertungen durchgeführt. Die entsprechenden statistischen Testmethoden geben wir zur besseren Übersicht in den jeweiligen Ergebnisbesprechungen an. Als Signifikanzlevel nahmen wir den Wert $\mathrm{p}<0,05$ an.

\subsection{Ergebnisse und Diskussion}

Unsere Umfrage wurde insgesamt 540-mal aufgerufen und der Fragenkatalog 169-mal vollständig ausgefüllt zurückgeschickt. Unvollständige Fragebögen wurden von der Auswertung ausgeschlossen. Die geographische Verteilung der Antwortenden mit vollständig ausgefüllten Fragebögen wurde bereits zuvor als relativ ausgeglichen beschrieben (Fritze et al. 2019; Online Supplement www.nulonline.de Webcode 2231). Die Mehrzahl der Teilnehmer*innen waren Behördenvertreter*innen und Fachgutachter*innen. In absteigender Anzahl nahmen Vertreter*innen von Umweltschutzorganisationen und des Windenergiesektors sowie Wissenschaftler*innen an der Umfrage teil.

\subsubsection{Aktivität und Artvorkommen: Beurteilung von Erfassungsmethoden in Abhängigkeit von Saison und Habitat}

Eine grundlegende Beschreibung der verfügbaren Methoden zur Erfassung der Aktivität und Artenvielfalt von Fledermäusen im Rahmen von Windkraftvorhaben 
findet sich in Brinkmann et al. (2011). Prinzipiell gelten Detektorbegehungen (manuelle bioakustische Erfassung), automatische bioakustische Aufnahmen in Bodennähe und in Gondelhöhe (Gondelmessung) sowie Netzfänge von Fledermäusen als etablierte Methoden.

Um die Aktivität von Fledermäusen zu erfassen, erachteten die Behördenvertreter*innen die Gondelmessung als am besten geeignet (4 Punkte; Abb. 3.1). Zur Erfassung der Artenvielfalt erhielt die Gondelmessung insbesondere während der Migrationszeit im Offenland gute Bewertungen (4 Punkte). Behördenvertreter*innen beurteilten die automatische Erfassung in Bodennähe und Detektorbegehungen unabhängig von Saison und Habitat als mittelmäßig geeignet, um Aktivität und Artenvielfalt von Fledermäusen lokal zu erfassen (3 Punkte, Anhang Frage 14 bis 17). Fachgutachter*innen bewerteten die verschiedenen akustischen Methoden ähnlich wie die Behördenvertreter*innen, wobei sie den automatischen Erfassungsmethoden eine bessere Eignung zuschrieben. Vertreter*innen von Umweltschutzorganisationen bewerteten die akustischen Methoden ähnlich wie die Behördenvertreter*innen. Alle Fachexpert*innen hielten den Netzfang für wenig geeignet, um Aktivitäten von Fledermäusen zu ermitteln.

- Behördenvertreter $(n=73)$

A) Aktivităt

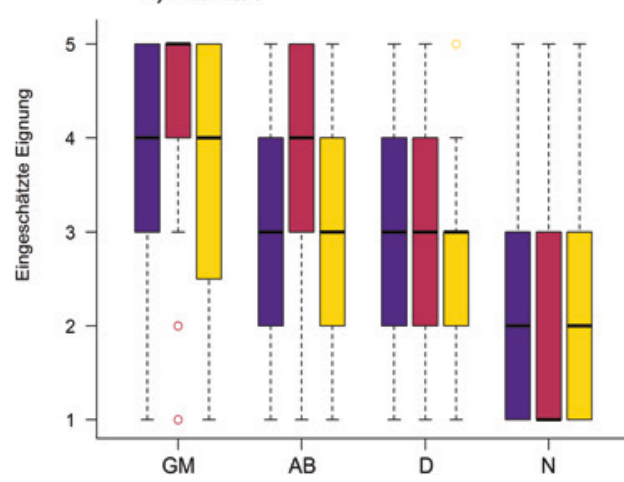

$\square$ Vertreter einer Umweltschutzorganisation $(n=21)$

B) Artenvielfalt

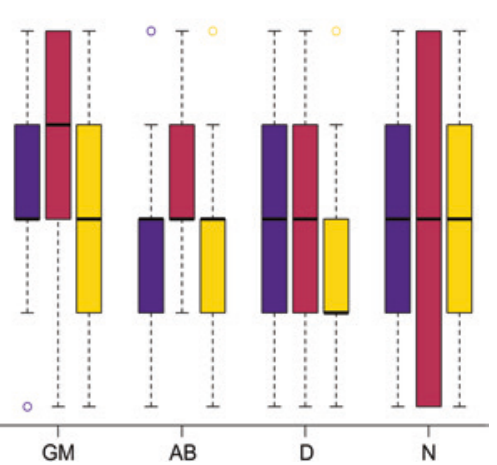

Abb. 3.1 Einschätzung der Eignung ( $1=$ sehr schlecht, $5=$ sehr gut $)$ verschiedener Methoden durch Fachexpert*innen um Aktivität (a) und Artenvielfalt (b) zu erfassen. GM=Gondelmessung, $\mathrm{AB}=$ automatische akustische Erfassung in Bodennähe, $\mathrm{D}=$ Detektorbegehung, $\mathrm{N}=$ Netzfang. Schwarze horizontale Linien entsprechen den Medianwerten, Boxen umfassen $50 \%$ der Datenpunkte

Fig. 3.1 Assessment of the suitability $(1=$ very bad, $5=$ very good $)$ of several methods by expert groups to document activity (a) and species diversity (b). GM=automated acoustic monitoring at nacelle height, $\mathrm{AB}=$ automated acoustic monitoring at ground level, $\mathrm{D}=$ acoustic monitoring via transects, $\mathrm{N}=$ mist-netting. Behördenvertreter $=$ members of conservation authorities, Fachgutachter $=$ consultants, Vertreter einer Umweltschutzorganisation $=$ member of an NGO. Black horizontal lines represent median values, boxes encompass $50 \%$ of the data points 
Die Fachexpert*innen bewerteten die akustische Erfassung zur Dokumentation der lokalen Artenvielfalt und der Aktivität geeignet (Abb. 3.1). Dem Netzfang wurde jedoch eine höhere Wertigkeit zur Erfassung der lokalen Artenvielfalt zugesprochen. Vor allem für Waldstandorte wurde der Netzfang positiv bewertet (Abb. 3.2; Anhang Frage 14 bis 17). Besonders für den Zeitraum der Wochenstubenphase erachteten Behördenvertreter*innen und Fachgutachter*innen den Netzfang im Wald als gut bzw. sehr gut geeignet. Diese Einschätzung deckt sich mit den Empfehlungen, die einige Bundesländer für Waldstandorte abgaben (Hurst et al. 2015). Auffällig unterschiedlich bewerteten Behördenvertreter*innen und Fachgutachter*innen die Eignung von Netzfängen während der Wochenstubenzeit an Offenlandstandorten. Während die Behördenvertreter*innen hier eine mittelmäßige Eignung des Netzfangs sahen, waren die Fachgutachter*innen diesbezüglich eher kritisch. Vertreter*innen von Umweltschutzorganisationen bewerteten den Netzfang durchgehend und unabhängig vom Standort als eher mittelmäßig geeignet. Alle Fachexpert*innen stimmten überein, dass Netzfänge

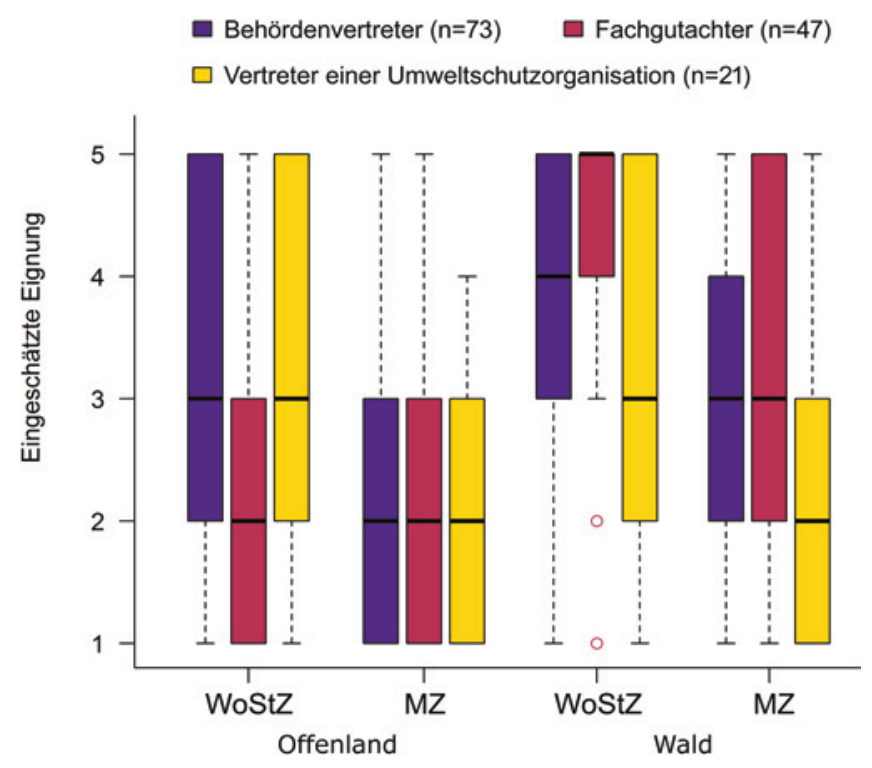

Abb. 3.2 Bewertung der Eignung ( $1=$ sehr schlecht, $5=$ sehr gut) des Netzfangs zur Erfassung der Artenvielfalt in Offenland und Wald während der Wochenstubenzeit (WoStZ) und der Migrationzeit (MZ) durch verschiedene Expert*innengruppen. Schwarze horizontale Linien entsprechen den Medianwerten, Boxen umfassen $50 \%$ der Datenpunkte

Fig. 3.2 Assessment of the suitability ( $1=$ very bad, $5=$ very good $)$ of mist-netting to document species richness in non-forest (Offenland) and forest sites (Wald) during (WoStZ) and outside the maternity period (MZ) by several expert groups. Behördenvertreter = members of conservation authorities, Fachgutachter $=$ consultants, Vertreter einer Umweltschutzorganisation $=$ member of an NGO Black horizontal lines represent median values, boxes encompass $50 \%$ of the data points 
eher schlecht geeignet sind, um im Offenlandbereich die Artenvielfalt zur Migrationszeit zu erfassen.

Da die Gondelmessung von allen Fachexpert*innen als überdurchschnittlich geeignet bewertet wurde (Abb. 3.1), betrachteten wir zusätzlich die Bewertung der Praxistauglichkeit. In dieser Auswertung wurden Behördenvertreter*innen $(n=48)$ und Gutachter*innen $(\mathrm{n}=42)$ berücksichtigt, die bereits sowohl Gondelmessungen im Offenland als auch im Wald bezüglich ihrer Eignung und Praxistauglichkeit bewertet hatten. Um beides gegenüberzustellen, bildeten wir den folgenden Index: (Praxistauglichkeit - Eignung)/4. Da sich dieser für Wald und Offenland nicht unterschied (Wilcoxon-Rangsummentest, Behördenvertreter*innen: $\mathrm{W}=1149$, $\mathrm{p}=0,98$; Fachgutachter*innen: $\mathrm{W}=834, \mathrm{p}=0,60$ ), fassten wir die Werte für beide Lebensstätten zusammen. Obgleich ein Großteil der jeweiligen Expert*innen die Gondelmessung zur Aktivitätserfassung als gleichermaßen praxistauglich wie geeignet bewertete (BehöV 52\%, FachG $64 \%$; Abb. 3.3), ergab die statistische Untersuchung Unterschiede. Die Praxistauglichkeit wurde insgesamt signifikant geringer bewertet als die Eignung (Wilcoxon-Vorzeichen-Rang-Test, Behördenvertreter*innen: $V=207, p=0,04$; Fachgutachter*innen: $V=106, p=0,01 ;$ Abb. 3.3).

Die Fachexpert*innen bewerteten die Eignung und die Praxistauglichkeit der Gondelmessung für beide Habitate gleich (Wilcoxon-Rangsummentest, Behördenvertreter*innen: $\mathrm{W}=1149, \mathrm{p}=0,98$; Fachgutachter*innen: $\mathrm{W}=834, \mathrm{p}=0,60$ ). Etwa die Hälfte der Behördenvertreter*innen bewertete die Gondelmessung zur Aktivitätserfassung von Fledermäusen als ebenso geeignet wie praxistauglich. Bei den Fachgutachter*innen lag der Wert bei $64 \%$ (Median-Indexwert=0; Abb. 3.3). Dennoch zeigte sich, dass die Praxistauglichkeit von beiden Fachexpert*innengruppen insgesamt signifikant geringer als die Eignung eingeschätzt wurde (Wilcoxon-Vorzeichen-Rang-Test, Behördenvertreter*innen: $\mathrm{V}=207, \mathrm{p}=0,04$; Fachgutachter*innen: $\mathrm{V}=106, \mathrm{p}=0,01)$.

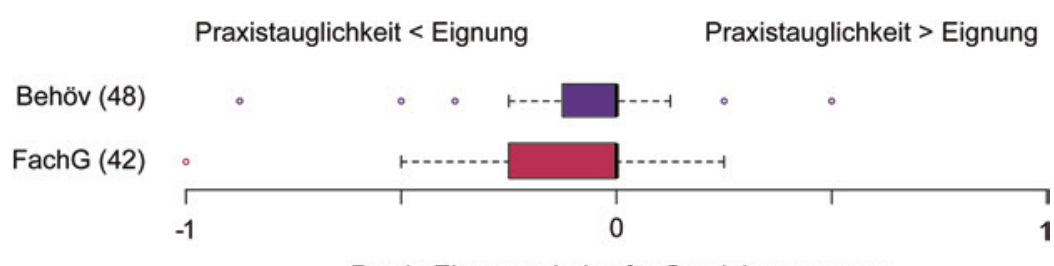

Praxis-Eingungs-Index für Gondelmessungen

\begin{abstract}
Abb. 3.3 Einschätzung von Praxistauglichkeit und Eignung der Gondelmessung zur Aktivitätserfassung durch Behördenvertreter*innen (Behöv) und Fachgutachter*innen (FachG). Der Praxis-Eignungs-Index errechnet sich aus (Praxistauglichkeit - Eignung)/4. Negative Werte bedeuten, dass die Praxistauglichkeit vergleichsweise gering eingeschätzt wird. Schwarze vertikale Linien entsprechen den Medianwerten, Boxen umfassen $50 \%$ der Datenpunkte
\end{abstract}

Fig. 3.3 Assessment of the practicability and suitability of automated acoustic monitoring of bats at nacelle heights to monitor overall bat activity by members of conservation authorities (Behöv) and by consultants (FachG). The index calculates as: (rank of practicability - rank of suitability)/4. Negative values indicate that practicability was considered to be relatively low. Black vertical lines represent median values, boxes encompass $50 \%$ of the data points 
Über die Eignung von Gondelmessungen zur Erfassung der akustischen Aktivität im Gefahrenbereich von WEA gibt es in der Literatur ein uneinheitliches Bild. Einerseits wird dargelegt, dass die erfassten Aktivitäten im Gondelbereich sich nicht signifikant zwischen Offenland- und Waldstandorten unterscheiden (Reers et al. 2016). Andererseits wird auf die Problematik hingewiesen, dass sich insbesondere an Waldstandorten Fledermäuse entlang des Turms in den Gefahrenbereich der Rotorblätter bewegen könnten, ohne von einer akustischen Erfassung in Gondelhöhe registriert zu werden (Hurst et al. 2015). Hurst et al. (Kap. 2) sowie Bach et al. (Kap. 4) empfehlen daher die Anbringung eines zweiten Mikrofons unterhalb der tiefsten Stelle des Rotoroperationskreises. Die relativ geringe Reichweite des Mikrofons ist je nach Fledermausart eines der generellen Probleme der bioakustischen Erfassung in der Gondel (Lindemann et al. 2018; Kap. 1). Die Aufnahmen im unteren Rotorbereich vergrößern die Detektionsreichweite. Des Weiteren können Fledermäuse, die sich dem Rotorenbereich von unten her nähern, überhaupt erst erfasst werden, denn diese würden bei laufenden WEA den Detektionsbereich des Mikrofons in der Gondel gar nicht erst erreichen. Es ist bekannt, dass sich Insekten an den WEA versammeln (Long et al. 2011; Trieb 2018). Diese könnten von sogenannten „Gleanern“, wie Langohren (Plecotus spp.) und Fransenfledermäusen (Myotis nattereri) vom Mast abgesammelt werden. Ein weiteres Problem besteht darin, dass manche Arten nur schwer anhand von Echoortungsrufen $\mathrm{zu}$ unterscheiden sind und automatische Identifizierungsprogramme fehleranfällig sind (Russo und Voigt 2016; Rydell et al. 2017).

\subsubsection{Radiotelemetrie als Methode der Habitatnutzungsanalyse}

Auf die Frage, ob Radiotelemetrie unter bestimmten Gesichtspunkten zwingend erforderlich sei, zeigten die Fachexpert*innengruppen deutliche Unterschiede in ihren Beurteilungen (Abb. 3.4; Anhang Frage 18). Während sich Behördenvertreter*innen und Fachgutachter*innen einig waren, dass die Radiotelemetrie für keine Art im Offenland notwendig sei, befürworteten die Behördenvertreter*innen die Radiotelemetrie von besonders schlaggefährdeten Arten im Offenland häufiger als die Fachgutachter*innen (ungefähr $60 \%$ gegenüber ungefähr $40 \%$ ). Weitgehend unabhängig von der Schlaggefährdung erachteten die meisten Vertreter*innen von Umweltschutzorganisation die Radiotelemetrie im Offenland als erforderlich.

Insgesamt war die Zustimmung zum Einsatz von Radiotelemetrie im Wald bei allen Fachexpert*innen weitaus größer als für Standorte im Offenland. Dies deckt sich mit den Empfehlungen der Länderleitfäden für Waldstandorte nach Hurst et al. (2015). Über $70 \%$ der Behördenvertreter*innen und Fachgutachter*innen hielten die Radiotelemetrie aller Arten im Wald für erforderlich, bei besonders schlaggefährdeten Arten sogar über $85 \%$ der jeweiligen Fachexpert*innengruppe. Auch von Vertreter*innen von Umweltschutzorganisationen wurde die Radiotelemetrie im Wald, besonders bei schlaggefährdeten Arten, stark befürwortet. Generell waren 


\section{Radiotelemetrie}

Raumnutzungsanalyse

Quartiersuche
Raumnutzungsanalyse \& Quartiersuche

Nie $\square$ Keine Beurteilung alle Arten
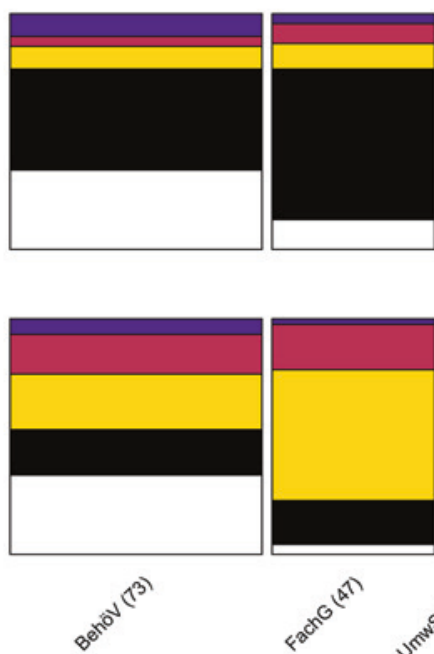
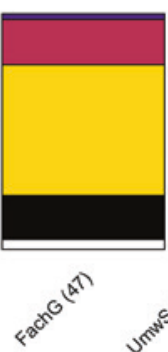

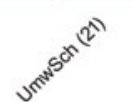

schlaggefährdete Arten

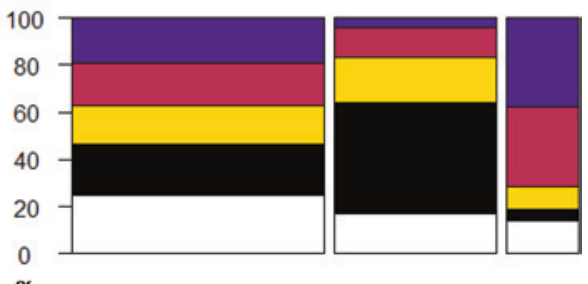

$\%$
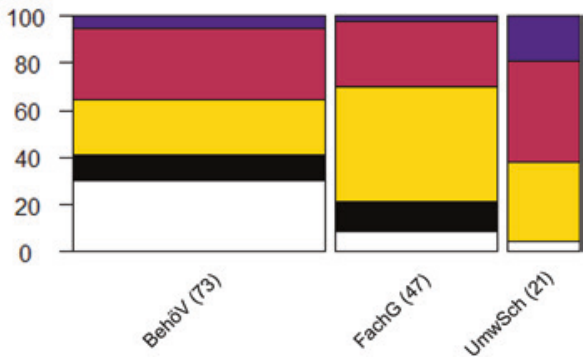

Abb. 3.4 Antworten der Fachexpert*innen auf die Frage, für welche Erfassungsaufgaben Radiotelemetriestudien zwingend Teil von Genehmigungsverfahren sein sollten. Die Breite der Balkensäule steht in Relation zu der Anzahl der Antworten der jeweiligen Expertengruppe (BehöV=Behördenvertreter*innen, FachG $=$ Fachgutachter*innen, UmwSch $=$ Vertreter*innen einer Umweltschutzorganisation)

Fig. 3.4 Answers of expert groups regarding the question: When should radiotracking studies be mandatory during environmental impact assessments during the planning process. The width of the bar indicates the relative number of answers for the specific expert group (BehöV $=$ members of the conservation authorities, $\mathrm{FachG}=$ consultants, $\mathrm{UmwSch}=$ members of an environmental NGO)

Behördenvertreter*innen bei der Bewertung der Radiotelemetrie als zwingend erforderliche Maßnahme zurückhaltender als die anderen Expert*innengruppen; sie gaben bei dieser Frage am häufigsten keine Beurteilung ab.

$27 \%$ der Befragten kommentierten die Frage bezüglich der Notwendigkeit von Radiotelemetrie. Es wurden Bedenken zur begrenzten Aussagekraft von Radiotelemetriestudien zum Ausdruck gebracht (9\% aller Befragten). Kritikpunkte waren hier hauptsächlich zu kurze Untersuchungszeiträume und zu geringe Zahl an telemetrierten Tieren und somit fehlende Repräsentanz. Die Wichtigkeit der Radiotelemetrie zur Erfassung der Lebensstätten speziell kleinräumig jagender, waldlebender Arten wurde über Kommentare durch $7 \%$ der Befragten hervorgehoben. $4 \%$ der Befragten äußerten tierschutzrechtliche Bedenken angesichts der als gering eingeschätzten Aussagekraft der Radiotelemetrie. 


\title{
3.3.3 Gondelmonitoring und Schlagopfersuchen
}

Fachgutachter*innen und Windenergievertreter*innen gaben jeweils zu etwa drei Viertel an, dass sie an Schlagopfersuchen oder Gondelmonitorings beteiligt waren; die meisten davon an beiden Maßnahmen (Abb. 3.5). Von den Behördenvertreter*innen waren ungefähr die Hälfte bereits in solche betriebsbegleitenden Monitoringmaßnahmen involviert; zumeist im Rahmen von Stellungnahmen. Vergleichsweise wenige Wissenschaftler*innen und Vertreter*innen von Umweltschutzorganisationen hatten praktische Erfahrung im betriebsbegleitenden Monitoring (Abb. 3.5; Anhang Frage 22). Bemerkenswert ist der hohe Anteil an Fachgutachter*innen und Behördenvertreter*innen, die keine Erfahrung mit den beiden Methoden hatten.

\section{Beteiligung an betriebsbegleitenden Monitoringmaßnahmen im Rahmen von:}

Gondelmessungen \& Schlagopfersuchen nur Gondelmessung nur Schlagopfersuchen
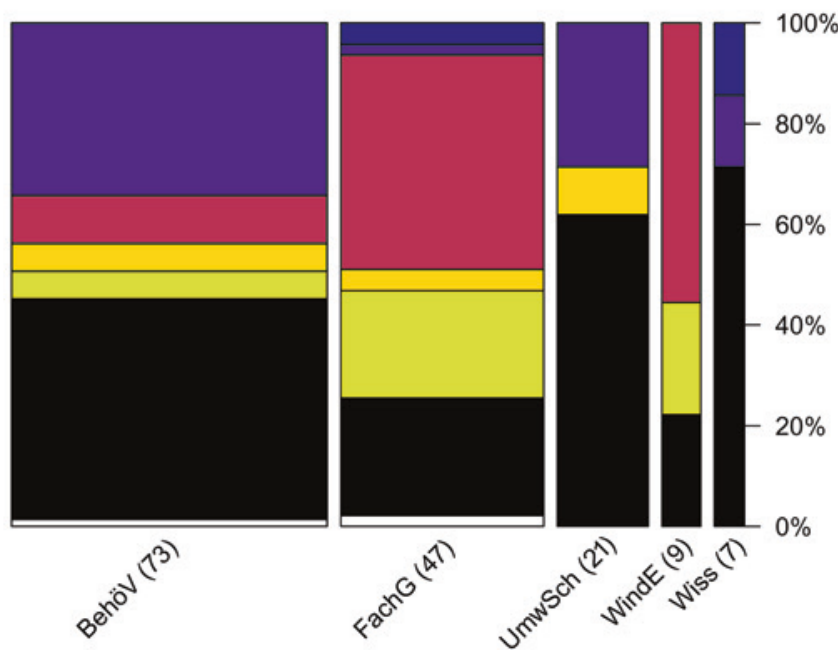

\begin{abstract}
Abb. 3.5 Teilnahme der Fachexpert*innen an betriebsbegleitenden Monitoringmaßnahmen. Die Breite der Balkensäule steht in Relation zu der Anzahl der Antworten der jeweiligen Expertengruppe $\quad(B e h o ̈ V=$ Behördenvertreter*innen, $\quad F a c h G=$ Fachgutachter*innen, UmwSch $=$ Vertreter*innen einer Umweltschutzorganisation, WindE $=$ Vertreter*innen des Windenergiesektors, Wiss $=$ Wissenschaftler*innen)
\end{abstract}

Fig. 3.5 Involvement of experts in environmental impact assessments of operating wind turbines. The width of the bar indicates the relative number of answers for the specific expert group (BehöV $=$ members of the conservation authorities, $F a c h G=$ consultants, $\mathrm{UmwSch}=$ members of an environmental NGO) 


\subsubsection{Diskrepanz zwischen akustischer Aktivität in Gondelhöhe und geschätzter Schlagopferzahl}

Zwischen den Fachexpert*innengruppen herrschte Uneinigkeit darüber, inwieweit die auf den Schlagopfersuchen basierende Schlagopferhochrechnung mit der bioakustischen Aktivitätsmessung auf Gondelhöhe korrelierte (Anhang Frage 23 und 24). Gutachter*innen gaben signifikant öfter als Behördenvertreter*innen an, bereits Diskrepanzen festgestellt zu haben (Fachgutachter*innen 59 \%, Behördenvertreter*innen $27 \%, \mathrm{Chi}^{2}$-Test: $\left.\mathrm{n}_{\text {total }}=52, \mathrm{p}=0,04\right)$.

Betrachtet man nur die Fachgutachter*innen, die Diskrepanzen zwischen Schlagopferzahlen und Gondelmessungen am selben Standort feststellten, ergibt sich kein klarer Trend. Die meisten Befragten gaben an, sowohl mit Fällen konfrontiert gewesen zu sein, in denen im Vergleich zur Gondelmessung relativ wenige, als auch mit Fällen, bei denen relativ viele Schlagopfer verzeichnet

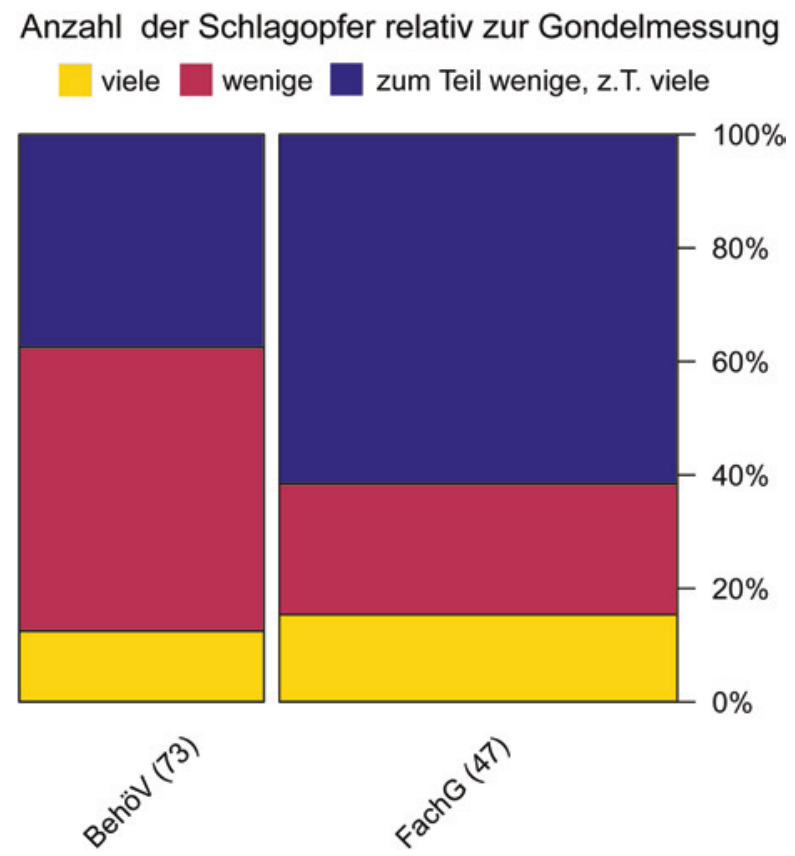

Abb. 3.6 Bewertung der Anzahl der gefundenen Schlagopfer in Relation zur Gondelmessung. Die Kategorien „viele“ und „wenige“ sind ein subjektives Maß für die Unter- bzw. Überschätzung der Schlagopferzahl an WEA. Die Breite der Balkensäule steht in Relation zu der Anzahl der Antworten der jeweiligen Fachexpert*innengruppe (BehöV=Behördenvertreter*innen, $\mathrm{FachG}=$ Fachgutachter*innen)

Fig. 3.6 Assessment of the number of observed bats killed at wind turbines in relation to the acoustic activity of bats at nacelle height. The cagetories 'viele' (more) and 'wenige' (less) is a personal evaluation of the under or overestimation of the number of fatalities at wind turbines. The width of the bar indicates the relative number of answers for the specific expert group (BehöV $=$ members of the conservation authorities, $\mathrm{FachG}=$ consultants) 
wurden (Abb. 3.6). Vermutlich gibt es Standorte, wie zum Beispiel in Wäldern, wo die Schlagopfersuche erhebliche Schwierigkeiten bereiten könnte, sodass diese nicht notwendigerweise verlässliche Daten abwirft. Daraus lässt sich ableiten, dass weder die eine noch die andere Methode allein eine verlässliche Einschätzung des Tötungsrisikos zulässt.

Auf die Frage, wie realistisch die Zahlen aus der Schlagopferhochrechnung nach Korner-Nievergelt et al. (2011) sind, gab etwa die Hälfte der Befragten an, es entweder nicht einschätzen zu können (36\%) oder die Hochrechnung generell nicht für sinnvoll zu halten (15\%). Die übrigen Personen waren geteilter Meinung darüber, ob die errechneten Zahlen realistisch sind (24\% realistisch, $26 \%$ unrealistisch). Mit etwa $40 \%$ gaben Behördenvertreter*innen am häufigsten an, keine Einschätzung zur Schlagopferhochrechnung vornehmen zu können. Nur etwa jeder fünfte Befragte gab an, dass die Schlagopferhochrechnung realistische Zahlen liefere (Abb. 3.7). Beinahe alle Befragten, die die Hochrechnung hinterfragten, schätzten die tatsächliche Zahl der Schlagopfer höher ein als die berechnete.

\section{Bewertungen der Schlagopferhochrechnungen und derer Ergebnisse \\ realistisch $\square$ unrealistisch, zu hoch unrealistisch, zu niedrig \\ $\square$ nicht sinnvoll $\square$ keine Einschätzung}
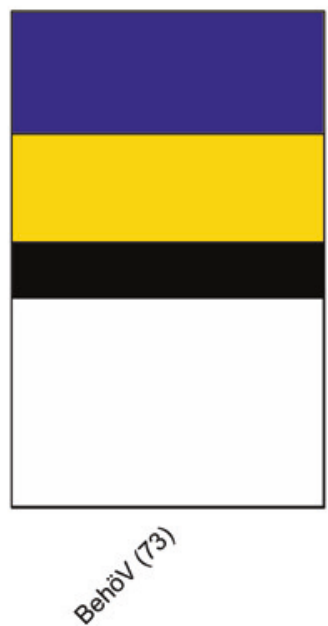
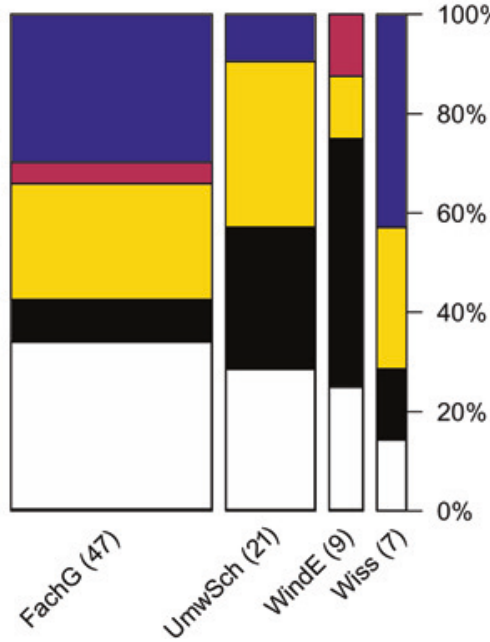

Abb. 3.7 Bewertung der Schlagopferhochrechnungen. Die Breite der Balkensäule steht in Relation zu der Anzahl der Antworten der jeweiligen Fachexpertengruppe (BehöV=Behördenvertreter*innen, FachG =Fachgutachter*innen, UmwSch = Vertreter*innen einer Umweltschutzorganisation, WindE $=$ Vertreter*innen des Windenergiesektors, Wiss $=$ Wissenschaftler*innen)

Fig. 3.7 Assessment of the estimated number of bats killed at wind turbines. The width of the bar indicates the relative number of answers for the specific expert group (BehöV $=$ members of the conservation authority, $\mathrm{FachG}=$ consultant, $\mathrm{UmwSch}=$ members of an environmental NGO, WindE $=$ member of a wind energy company, Wiss $=$ scientist $)$ 


\subsection{Zusammenfassende Empfehlungen zum Methodenrepertoire}

Zusammenfassend lässt sich festhalten, dass die Erfassung von Fledermäusen in Bodennähe mittels Netzfang und akustischer Detektorbegehung nach der Expert*inneneinschätzung eher für Wald- als für Offenlandstandorte geeignet ist, da die Erfassung am Boden offenbar nicht unbedingt die Aktivität und das Artvorkommen der Tiere im Bereich der Rotoren widerspiegelt. Die beiden bodengebundenen Methoden sind jedoch sinnvoll und notwendig, um vor dem Bau der WEA mögliche Beeinträchtigung von Lebensstätten festzustellen. Mit der Detektorbegehung können zum Beispiel Lebensstätten erkundet und identifiziert werden, in denen besonders viele Fledermäuse aktiv sind, um Hinweise auf Jagdhabitate und Wochenstuben zu erhalten. Mit dem Netzfang können vorkommende Fledermausarten einwandfrei bestimmt werden und durch die Ermittlung des Reproduktionstatus und Alters (Laktationsmerkmale, Juvenile) kann erkannt werden, ob sich Fledermäuse im Einzugsgebiet potenziell reproduzieren. Beide Methoden spielen deshalb im Wald eine herausragende Rolle, da Wälder wichtige Habitate für Fledermäuse darstellen, die durch die Rodung der WEA-Stellflächen und Zuwegungen in Mitleidenschaft gezogen werden können (Rodrigues et al. 2016). Auch die Radiotelemetrie spielt nach Auffassung der Umfrageteilnehmer*innen besonders im Wald eine Rolle. Über die Nachverfolgung laktierender Weibchen eignet sich die Methode besonders gut, um Wochenstuben ausfindig zu machen und deren Nähe zum WEA-Standort festzustellen. Dies ist vor allem deshalb von Bedeutung, weil die Streifgebiete vieler schlaggefährdeter Fledermausarten zur Wochenstubenzeit weit über einen Radius von $200 \mathrm{~m}$ um das Quartier herum reichen (z. B. Nyctalus noctula, Großer Abendsegler: Mackie und Racey 2007; Nyctalus leisleri, Kleinabendsegler: Shiel et al. 1999; Vespertilio murinus, Zweifarbfledermaus: Jaberg und Blant 2003). Auch sogenannte Raumnutzungsanalysen werden mittels Kreuzpeilung einzelner besenderter Tiere durchgeführt, um den Bewegungsradius der Tiere festzustellen und zu ermitteln, ob die Tiere den Bereich der geplanten WEA nutzen. Anzumerken ist hierbei jedoch, dass eine Raumnutzungsanalyse von zwei oder drei Individuen, wie es von einigen Behörden gefordert wird (MKULNV NRW 2017), eine nur sehr geringe Aussagefähigkeit hat, um auf das Verhalten aller Individuen einer Population im Gebiet zu schließen. Hinzu kommt, dass eine solche Raumnutzungstelemetrie nur eine Momentaufnahme ist und die Prognosesicherheit für das Verhalten der Tiere für die gesamte WEA-Laufzeit bedacht werden sollte. Die existierenden Leitfäden müssen daher dringend um die wissenschaftlichen Erkenntnisse der letzten 20 Jahre zum Raumnutzungsverhalten nachgebessert und entsprechend angepasst werden, was prioritär zu längeren Untersuchungszeiträumen führen würde.

Während des Betriebs von WEA stellen Gondelmessung und Schlagopfersuche wichtige Methoden dar, um das Schlagrisiko nach erteilter Genehmigung zu ermitteln bzw. die Wirksamkeit der Auflagen zu überprüfen und gegebenenfalls Abschaltzeiten anzupassen. Die Einschätzungen der befragten Fachexpert*innen, vor allem die der Fachgutachter*innen, legen nahe, dass Gondelmessung und 
Schlagopfersuche sich nicht gegenseitig ersetzen können, sondern sich vielmehr ergänzen. Über die Hälfte der Antworten der Fachgutachter*innen zeigt, dass Fledermausaktivitäten in Gondelhöhe nicht notwendigerweise die Zahlen aus der Schlagopfersuche widerspiegeln. Es scheint daher empfehlenswert, beide Methoden parallel in den Genehmigungsverfahren anzuwenden.

Das in den Umfrageergebnissen abgebildete Misstrauen gegenüber der Schlagopferhochrechnung resultiert vermutlich aus den Unsicherheiten dieser Methode, die sich aus der rechnerischen Berücksichtigung der Sucheffizienz nach Schlagopfern (Auffindbarkeit der Schlagopfer aufgrund der Beschaffenheit der Fläche, Vegetationsbedeckung, Größe der absuchbaren Fläche, Sucheffizienz des Suchenden, Kadaverabtrag durch Prädatoren) und der Kadaverabtragrate ergeben. Korner-Nievergelt et al. (2011; http://www.kollisionsopfersuche.uni-hannover. de/) etablierten ein inzwischen weitläufig genutztes System zur Abschätzung von Schlagopferzahlen basierend auf tatsächlichen Schlagopferfunden. Mithilfe dieses Onlinetools und dem zugehörigen Protokoll und der Anleitung ist es möglich, standardisierte Schlagopfersuchungen durchzuführen, die Einschränkungen zu dokumentieren und die Schlagopferzahl automatisch abzuschätzen. Die generelle Skepsis gegenüber derartigen Methoden resultiert unserer Auffassung nach aus der Komplexität des mathematischen Modells und der dennoch verbleibenden Unsicherheit der hochgerechneten Werte. Diese Unsicherheiten entsprechen jedoch stets der Größe der Einschränkungen. Wenn also die absuchbare Fläche nicht ausreichend groß ist (weil z. B. Ackerflächen inbegriffen sind) oder wenn nicht genug Begehungen stattgefunden haben, verbleibt eine große statistische Ungenauigkeit. Im Umkehrschluss kann man diese Unsicherheit verkleinern, indem die Suchbedingungen optimiert und die Anzahl der Begehungen vergrößert werden. Zudem berechnet die Formel von Korner-Nievergelt et al. (2011) ein Konfidenzintervall für das Ergebnis, das eine Abschätzung der Genauigkeit der Berechnung ermöglicht.

Behördenvertreter*innen müssen aufgrund geschätzter oder tatsächlich gefundener getöteter Individuen über Auflagen entscheiden. Es scheint deshalb ratsam, Schlagopfersuchen weiter zu beauflagen. Zudem ist eine Abschätzung anhand von akustischen Messungen nur sehr bedingt möglich bzw. kann nur durch speziell dafür entwickelte Programme, wie zum Beispiel Probat (Behr et al. 2018), erfolgen. Für einen flächendeckenden Einsatz solcher Systeme ist mehr Transparenz erforderlich, damit alternative Messmethoden, die ergänzende Informationen liefern, implementiert werden können und die Unsicherheit in der Gefahrenabschätzung effektiv reduziert wird.

Danksagung Wir danken den Umfrageteilnehmer*innen für die Zeit, die sie sich für die Beantwortung der Fragebögen genommen haben, sowie den verschiedenen Fachexpert*innen für die Diskussion der Ergebnisse. Wir bedanken uns bei den zwei anonymen Gutachtern für ihre konstruktive Kommentare.

Die Veröffentlichung wurde durch den Open-Access-Publikationsfonds für Monografien der Leibniz-Gemeinschaft gefördert. 


\section{Literatur}

Bach L (2001) Fledermäuse und Windenergienutzung - reale Probleme oder Einbildung? Vogelkundliche Ber aus Niedersachs 33:119-124

Baerwald EF, D'Amours GH, Klug BJ, Barclay MR (2008) Barotrauma is a significant cause of bat fatalities at wind turbines. Curr Biol 18:R695-R696

Behr O, Brinkmann R, Hochradel K, Mages J, Korner-Nievergelt F, Reinhard H, Simon R, Stiller F, Weber N, Nagy M (2018) Bestimmung des Kollisionsrisikos von Fledermäusen an Onshore-Windenergieanlagen in der Planungspraxis - Endbericht des Forschungsvorhabens gefördert durch das Bundesministerium für Wirtschaft und Energie Universität Erlangen-Nürnberg Lehrstuhl für Sensorik. Erlangen: $416 \mathrm{~S}$

Brinkmann R (2011) Entwicklung von Methoden zur Untersuchung und Reduktion des Kollisionsrisikos von Fledermäusen an Onshore-Windenergieanlagen: Ergebnisse eines Forschungsvorhabens 1. Aufl. Cuvillier, Göttingen: $457 \mathrm{~S}$

Brinkmann R, Behr O, Korner-Nievergelt F, Mages J, Niermann I, Reich M (2011) Entwicklung von Methoden zur Untersuchung und Reduktion des Kollisionsrisikos von Fledermäusen an Onshore-Windenergieanlagen. Cuvillier Verlag, Göttingen

Deutsche WindGuard (2019) Status des Windenergieausbaus an Land - Jahr 2018. www. windguard.de. Zugegriffen: 10. Apr. 2019

Dietz M, Krannich E, Weitzel M (2015) Arbeitshilfe zur Berücksichtigung des Fledermausschutzes bei der Genehmigung von Windenergieanlagen (WEA) in Thüringen Institut für Tierökologie und Naturbildung. Gonterskirchen: $121 \mathrm{~S}$

Dürr T (2002) Fledermäuse als Opfer von Windenergieanlagen in Deutschland. Nyctalus N.F 8:115-118

Dürr T, Bach L (2004) Fledermäuse als Schlagopfer von WEA - Stand der Erfahrungen mit Einblick in die bundesweite Fundkartei. Bremer Beitr Naturkunde Naturschutz 7:253-263

EEG (2017) Erneuerbare-Energien-Gesetz vom 21. Juli 2014 (BGBl. I S. 1066), das zuletzt durch Artikel 1 des Gesetzes vom 17. Juli 2017 (BGBl. I S. 2532) geändert worden ist

FA Wind (Fachagentur Windenergie an Land) (2018) Entwicklung der Windenergie im Wald Ausbau, planerische Vorgaben und Empfehlungen für Windenergiestandorte auf Waldflächen in den Bundesländern, Berlin: $44 \mathrm{~S}$

Fritze M, Lehnert LS, Heim O, Lindecke O, Roeleke M, Voigt CC (2019) Fledermaus im Schatten der Windenergie: Deutschlands Experten vermissen Transparenz und bundesweite Standards in den Genehmigungsverfahren. Naturschutz und Landschaftsplanung 51:20-27

Gebhard F, Kötteritzsch A, Lüttmann J, Kiefer A, Hendler R, Veith M (2016) Fördern Arbeitshilfen die Qualität von Fachgutachten? Eine Analyse von Fledermaus-Fachgutachten zur Planung von Windenergieanlagen. Naturschutz und Landschaftsplanung 48:177-183

Hurst J, Balzer S, Biedermann M, Dietz C, Dietz M, Höhne E, Karst I, Petermann R, Schorcht W, Steck C, Brinkmann R (2015) Erfassungsstandards für Fledermäuse bei Windkraftprojekten in Wäldern - Diskussion aktueller Empfehlungen der Bundesländer. Nat Landsch 90:157-169

Jaberg C, Blant J-D (2003) Spatio-temporal utilisation of roosts by the parti-coloured bat Vespertilio murinus L., 1758 in Switzerland. Mamm Biol 68:341-350

Korner-Nievergelt F, Korner-Nievergelt P, Behr O, Niermann I, Brinkmann R, Hellriegel B (2011) A new method to determine bird and bat fatality at wind energy turbines from carcass searches. Wildl Biol 17:350-363. https://doi.org/10.2981/10-121

Lehnert LS, Kramer-Schadt S, Schönborn S, Lindecke O, Niermann I, Voigt CC (2014) Wind farm facilities in Germany kill noctule bats from near and far. PloS one 9:e103106

Lindemann C, Runkel V, Kiefer A, Lukas A, Veith M (2018) Abschaltalgorithmen für Fledermäuse an Windenergieanlagen. Naturschutz Landschaftsplanung 50:418-425

Lintott PR, Richardson SM, Hosken DJ, Fensome SA, Mathews F (2016) Ecological impact assessments fail to reduce risk of bat casualties at wind farms. Curr Biol 26:R1135-R1136. https://doi.org/10.1016/j.cub.2016.10.003 
Long CV, Flint JA, Lepper PA (2011) Insect attraction to wind turbines: does colour play a role? European J Wildl Res 57:323-331. https://doi.org/10.1007/s10344-010-0432-7

Mackie IJ, Racey PA (2007) Habitat use varies with reproductive state in noctules bats (Nyctalus noctula): implications for conservation. Biol Conserv 140:70-77

MKULNV NRW (Ministerium für Klimaschutz, Umwelt, Landwirtschaft, Natur- und Verbraucherschutz des Landes Nordrhein-Westfalen) (2017) Leitfaden „Methodenhandbuch zur Artenschutzprüfung in Nordrhein-Westfalen -Bestandserfassung und Monitoring-“. Landesamt für Natur, Umwelt und Verbraucherschutz Nordrhein-Westfalen (LANUV). Recklinghausen: S.((http://artenschutz.naturschutzinformationen.nrw.de/artenschutz/))

MLUL (Ministerium für Landwirtschaft, Umwelt und Landwirtschaft) (2011) Beachtung naturschutzfachlicher Belange bei der Ausweisung von Windeignungsgebieten und bei der Genehmigung von Windenergieanlagen - Erlass des Ministeriums für Umwelt, Gesundheit und Verbraucherschutz (Windkrafterlass)

MULE (Ministerium für Umwelt, Landwirtschaft und Energie des Landes Sachsen-Anhalt) (2018) Leitfaden Artenschutz an Windenergieanlagen in Sachsen-Anhalt. Magdeburg: 47 S

Reers H, Hartmann S, Hurst J, Brinkmann R (2016) Bat activity at nacelle height over forest. Wind energy and wildlife Interactions (Köppel J). Springer, Cham, S 79-98

Rodrigues L, Bach L, Dubourg-Savage M-J, Karapandža B, Kovač D, Kervyn T, Dekker J, Kepel A, Bach P, Collins J, Harbusch C, Park K, Micevski B, Minderman J (2016) Leitfaden für die Berücksichtigung von Fledermäusen bei Windenergieprojekten Überarbeitung 2014. UNEP/ EUROBATS. Bonn: $146 \mathrm{~S}$

Russo D, Voigt CC (2016) The use of automated identification of bat echolocation calls in acoustic monitoring: a cautionary note for a sound analysis. Ecol Ind 66:598-602

Rydell J, Nyman S, Eklöf J, Jones G, Russo D (2017) Testing the performances of automated identification of bat echolocation calls: a request for prudence. Ecol Ind 78:416-420

Shiel C, Shiel R, Fairley J (1999) Seasonal changes in the foraging behaviour of Leisler's bats (Nyctalus leisleri) in Ireland as revealed by radio-telemetry. J Zool 249:347-358

Trieb F (2018) Interference of Flying Insects and Wind Parks (FliWip). Study Report. Deutsches Zentrum für Luft- und Raumfahrt: $30 \mathrm{~S}$

Voigt CC (2016) Fledermäuse und Windenergieanlagen: ein ungelöstes 'green-green' Dilemma. S. 43 in BfN-Skripten 432. Korn, H., Bockmühl, K. \& Schliep, R. (Hrsg) Biodiversität und Klima - Vernetzung der Akteure in Deutschland XII - Dokumentation der 12. Tagung

Voigt CC, Lehnert LS, Petersons G, Adorf F, Bach L (2015) Bat fatalities at wind turbines: German politics cross migratory bats. Eur J Wildl Res 61:213-219

Voigt CC, Lindecke O, Schönborn S, Kramer-Schadt S, Lehmann D (2016) Habitat use of migratory bats killed during autumn at wind turbines. Ecol Appl 26:771-783

Voigt CC, Popa-Lisseanu AG, Niermann I, Kramer-Schadt S (2012) The catchment area of wind farms for European bats: a plea for international regulations. Biol Conserv 153:80-88

Voigt CC, Straka TM, Fritze M (2019) Producing wind energy at the cost of biodiversity: a stakeholder view on a green-green dilemma. J Sustain Renew Energy 11:063303

Zahn A, Lustig A, Hammer M (2014) Potenzielle Auswirkungen von Windenergieanlagen auf Fledermauspopulationen. ANLiegen Nat 36:1-15 
Open Access Dieses Kapitel wird unter der Creative Commons Namensnennung 4.0 International Lizenz (http://creativecommons.org/licenses/by/4.0/deed.de) veröffentlicht, welche die Nutzung, Vervielfältigung, Bearbeitung, Verbreitung und Wiedergabe in jeglichem Medium und Format erlaubt, sofern Sie den/die ursprünglichen Autor(en) und die Quelle ordnungsgemäß nennen, einen Link zur Creative Commons Lizenz beifügen und angeben, ob Änderungen vorgenommen wurden.

Die in diesem Kapitel enthaltenen Bilder und sonstiges Drittmaterial unterliegen ebenfalls der genannten Creative Commons Lizenz, sofern sich aus der Abbildungslegende nichts anderes ergibt. Sofern das betreffende Material nicht unter der genannten Creative Commons Lizenz steht und die betreffende Handlung nicht nach gesetzlichen Vorschriften erlaubt ist, ist für die oben aufgeführten Weiterverwendungen des Materials die Einwilligung des jeweiligen Rechteinhabers einzuholen. 